\title{
Improving Accuracy of Tay Sachs Carrier Screening of the Non-Jewish Population: Analysis of 34 Carriers and Six Late-Onset Patients With HEXA Enzyme and DNA Sequence Analysis
}

\author{
NOH JIN PARK, CRAIG MORGAN, RAJESH SHARMA, YUANYIN LI, RAYNAH M. LOBO, JOY B. REDMAN, \\ DENISE SALAZAR, WEIMIN SUN, JULIE A. NEIDICH, AND CHARLES M. STROM \\ Molecular Genetics Department [N.J.P., Y.L., J.B.R., W.S., C.M.S.] and Biochemical Genetics Department [C.M., R.S., R.M.L., D.S., \\ J.A.N., C.M.S.], Quest Diagnostics Nichols Institute, San Juan Capistrano, California 92690
}

\begin{abstract}
The purpose of this study was to determine whether combining different testing modalities namely $\beta$-hexosaminidase A (HEXA) enzyme analysis, HEXA DNA common mutation assay, and HEXA gene sequencing could improve the sensitivity for carrier detection in non-Ashkenazi (AJ) individuals. We performed a HEXA gene sequencing assay, a HEXA DNA common mutation assay, and a HEXA enzyme assay on 34 self-reported Tay-Sachs disease (TSD) carriers, six late-onset patients with TSD, and one pseudodeficiency allele carrier. Sensitivity of TSD carrier detection was $91 \%$ for gene sequencing compared with $91 \%$ for the enzyme assay and $52 \%$ for the DNA mutation assay. Gene sequencing combined with enzyme testing had the highest sensitivity (100\%) for carrier detection. Gene sequencing detected four novel mutations, three of which are predicted to be disease causing [118.delT, 965A $\rightarrow \mathrm{T}(\mathrm{D} 322 \mathrm{~V})$, and $775 \mathrm{~A} \rightarrow \mathrm{G}$ (T259A)]. Gene sequencing is useful in identifying rare mutations in patients with TSD and their families, in evaluating spouses of known carriers for TSD who have indeterminate enzyme analysis and negative for common mutation analysis, and in resolving ambiguous enzyme testing results. (Pediatr Res 67: 217-220, 2010)
\end{abstract}

$\mathrm{T}$ ay-Sachs disease (TSD) is an autosomal recessive, neurodegenerative disorder caused by insufficient $\beta$-hexosaminidase A (HEXA) activity, leading to excess storage of $\mathrm{G}_{\mathrm{M} 2}$ ganglioside. Patients with the classical infant form manifest after the first few months of life. Symptoms include a cherry red spot on the fovea centralis of the macula, increased startle response, hypotonia, progressive weakness and loss of motor skills, seizures, and dementia. Death typically occurs between the ages of 2 and $5 \mathrm{y}$. Delayed onset (late infant, juvenile, or adult) forms occur less frequently; the later the onset, the less severe the disease. High-risk populations include Ashkenazi Jews (carrier frequency $\sim 1 / 30$ ), French Canadians, Cajuns, and Pennsylvania Dutch. Carrier frequency in other populations is $1 / 300$.

In the United States and Canada, carrier screening in the Ashkenazi Jewish population, instituted in the early 1970s, has resulted in a $90 \%$ decrease in incidence (three to five cases annually versus $\sim 60$ ) (1). Owing to this success, almost all newly diagnosed cases now have at least one parent of non-Ashkenazi Jewish descent. Thus, the American College of Obstetricians and

Received July 30, 2009; accepted September 26, 2009.

Correspondence: Charles M. Strom, M.D., Ph.D., Molecular Genetics Department, Quest Diagnostics Nichols Institute, 33608 Ortega Highway, San Juan Capistrano, CA 92690; e-mail: charles.m.strom@questdiagnostics.com

Supported by Quest Diagnostics Inc.
Gynecologists recommends carrier screening for couples of Ashkenazi Jewish, French-Canadian, and Cajun descent as well as for couples in whom only one member is at high risk based on ethnicity (2). Such screening is currently based on biochemical analysis of HEXA activity supplemented with molecular analysis for detection of the five most common HEXA gene mutations and two pseudodeficiency alleles associated with false-positive enzyme test results.

The biochemical assay often uses an uncharged, artificial substrate, 4-methlyumbelliferyl- $\beta$ - $N$-acetyl glucosaminide (MUG), to measure total hexosaminidase activity in serum (3). Total hexosaminidase is comprised of HEXA and hexosaminidase B (HEXB). HEXA is a heterodimer of alpha and beta chains, whereas HEXB is a homodimer of beta chains. In the biochemical assay, HEXB activity is measured after heat inactivation of the HEXA in a second serum aliquot. HEXA activity is then calculated as the difference between the total hexosaminidase and HEXB activities. Results are expressed as the percentage of HEXA activity relative to the total hexosaminidase activity.

Although the biochemical assay is reasonably accurate and cost effective, it does have several limitations. First, there is an indeterminate (gray-zone) range in which results from carriers and noncarriers overlap. As many as $10 \%$ of samples can have results in this range (4). Second, false-positive results occur in the serum of pregnant women and those taking oral contraceptives owing to the presence of another form of hexosaminidase $\mathrm{P}$ (i.e., HEXP) $(5,6)$. HEXP, which is not heat labile, reduces the calculated percentage of HEXA, and the woman may be incorrectly classified as a carrier. To circumvent this problem, the assay can be performed using white blood cells, which lack HEXP. Third, false-positive results (2\% in Ashkenazi Jewish, $35 \%$ in other populations) occur in individuals who carry a pseudodeficiency allele $(7,8)$. There are two such alleles: $739 \mathrm{C}>\mathrm{T}$, also termed ARG247TRP (R247W), and 745C $>\mathrm{T}$, also designated as ARG249TRP (R249W). These alleles code for HEXA enzymes with reduced activity against the artificial MUG substrate but not against the cellular $\mathrm{G}_{\mathrm{M} 2}$ ganglioside. Thus, biochemical assay

\footnotetext{
Abbreviations: HEXA, $\beta$-hexosaminidase A; HEXB, hexosaminidase B; HEXP, hexosaminidase P; MUG, 4-methlyumbelliferyl- $\beta$ - $N$-acetyl glucosaminide; TSD, Tay-Sachs Disease
} 
results are likely to be consistent with a TSD carrier, though the phenotype is benign. Fourth, a B1 variant phenotype can result in a false-negative test (individual classified as normal rather than as a carrier) $(9,10)$. Five $H E X A$ mutations have been identified that cause this phenotype. Their protein product has reduced activity against cellular $\mathrm{G}_{\mathrm{M} 2}$ ganglioside, but normal activity against the artificial MUG substrate. Fifth, a rare thermolabile HEXB can result in an apparent increase in HEXA activity, potentially masking a TSD carrier. Finally, the biochemical assay cannot differentiate carriers of the milder juvenile/adult mutations from carriers of the more severe, infantile mutations.

Three mutations account for $92-98 \%$ of carriers among Ashkenazi Jewish individuals $(11,12)$. The most common mutation is a TATC insertion at exon 11 (1278insTATC). A G to C transversion at the $5^{\prime}$ splice site in intron $12(1421+1 \mathrm{G} \rightarrow \mathrm{C}$, also designated +1 IVS12 $\mathrm{G} \rightarrow \mathrm{C}$ ) is the second most common mutation, and a glycine to serine mutation in exon $7(805 \mathrm{G} \rightarrow \mathrm{A}$, also known as G269S) is the third. The 1278insTATC and the G269S mutations are also commonly found in non-Ashkenazi Jewish populations, along with an intron 9 splice site mutation $(1073+1 \mathrm{G}>\mathrm{A}$, also termed +1 IVS9 G>A) and a $7.6 \mathrm{~kb}$ deletion (7.6-KB DEL, EX1). These five mutations, along with the two pseudodeficiency mutations discussed earlier, make up our standard HEXA DNA common mutation test used for carrier screening and diagnosis. Although such DNA testing is preferable to the enzyme assay in the Ashkenazi Jewish population (4), it would only detect $\sim 50 \%$ of carriers in other populations. The other $50 \%$ are attributed to nearly 100 disease-causing mutations present across the entire $H E X A$ gene. Therefore, enzyme analysis has remained the method of choice in the non-Ashkenazi Jewish population. An unanswered question is whether extensive DNA sequencing, encompassing all coding regions and splice junction sites, would be an appropriate alternative to either the enzyme analysis or the standard DNA mutation analysis in these populations. This is an important question as the annual TSD death rate has remained at $\sim 10$ for the last several decades, an increase of three to four over that in the Ashkenazi Jewish population in the United States and Canada. In this work, we examine the potential of $H E X A$ gene sequencing for carrier screening and diagnosis in a non-Ashkenazi Jewish population of purported TSD carriers.

\section{MATERIALS AND METHODS}

Patient information. Samples were obtained at the 2006 annual parents meeting of the National Tay-Sachs and Allied Diseases Association. Individuals were solicited who were either affected with TSD (all adult onset patients), self-reported parents of children or adults affected with TSD, or relatives of these index cases who have been told they were carriers of TSD. Obligate carrier status was known in only nine of the 33 (27\%) self-reported, unaffected carriers. Informed consent was obtained and samples were col- lected for both serum and blood/white blood cell preparations. This study was approved by the Western Institutional Review Board.

Study protocol. We performed the HEXA gene sequencing, HEXA DNA mutation test, and the HEXA enzyme assay on 34 self-reported TSD carriers, six late-onset patients with TSD, and one pseudodeficiency allele carrier. None of the carriers claimed to be of Ashkenazi Jewish descent, and none were pregnant at time of sample collection.

DNA extraction. DNA from most of the samples were extracted using Gentra Autopure LS (Minneapolis, MN) exactly as described by the manufacturer, and some samples were extracted using Qiagen Biorobot 9604 platform using MegAttract DNA blood M96 kit (Valencia, CA). Whole blood was used for DNA extraction in most cases, and in some cases, leukocytes were used. We observed no differences in sequencing quality between different extraction platforms and different starting materials.

HEXA gene sequencing. DNA sequencing and PCR were performed essentially as described by Huang et al. (13) except that DNA purification post-PCR was done using exonuclease (USB Corporation, Cleveland, $\mathrm{OH}$ )/ calf intestinal alkaline phosphatase (Promega, Madison, WI) digestion, and postdye terminator reaction DNA was purified by ethanol precipitation and resuspended in Hi-Di formamide (ABI, Foster City, CA). Fourteen amplicons, each amplifying one of 14 exons, were generated through individual PCR reactions. Amplification efficiency and correctness of sizes were verified by agarose gel electrophoresis. Sequencing covered exons 1 through 14 of chromosome 15 and at least 20-bp flanking sequences. Samples were resolved on an ABI 3730 automated DNA analyzer and data were analyzed using SeqScape software (both from Applied Biosystems).

HEXA DNA common mutation test. Mutation analysis was performed for five common mutations and two pseudoalleles: 1278insTATC; $1421+$ $1 \mathrm{G} \rightarrow \mathrm{C}(+1 \mathrm{IVS} 12 \mathrm{G} \rightarrow \mathrm{C}) ; 805 \mathrm{G} \rightarrow \mathrm{A}$ [GLY269SER $(\mathrm{G} 269 \mathrm{~S})$ ]; $1073+$ $1 \mathrm{G} \rightarrow \mathrm{A}(+1$ IVS9 $\mathrm{G} \rightarrow \mathrm{A}) ;$ 7.6-KB DEL, EX1; 739C $\rightarrow$ T [ARG247TRP $(\mathrm{R} 247 \mathrm{~W})]$; and 745C $\rightarrow \mathrm{T}$ [ARG249TRP (R249W)]. We used allele-specific primer extension to detect these mutations as per the method described by Strom et al. (14).

HEXA enzyme assay. The serum HEXA enzyme assay has been described previously (3). For this study, we set the normal HEXA activity level at $57-80 \%$ and the carrier/affected level at $0-51 \%$.

\section{RESULTS}

There was a single patient (male) who was determined to be a non-TSD carrier by all three testing modalities: Hex A enzyme analysis on serum, HEXA DNA common mutation assay, and HEXA gene sequencing. Therefore, this patient is unlikely to be a true carrier and will be eliminated from further discussion.

The HEXA gene sequencing assay detected two mutant alleles in six of the six adult-onset TSD-affected individuals. Thus, gene sequencing correctly classified $100 \%$ of TSD cases. Gene sequencing correctly classified 30 of $33(91 \%)$ TSD unaffected carriers. No mutation was detected in two of the remaining cases, and a novel mutation $(775 \mathrm{~A} \rightarrow \mathrm{G})$, predicted to be silent, was detected in the final case. Gene sequencing also successfully detected a pseudodeficiency mutation $[739 \mathrm{C} \rightarrow \mathrm{T}(\mathrm{R} 247 \mathrm{~W})]$ in the TSD pseudodeficiency carrier. Table 1 details the test results obtained from all three screening methods on the cases that were misclassified by gene sequencing. The average enzyme levels for late-onset TSD and TSD carriers were 10 and $43 \%$, respectively.

Table 1. Sequencing assay discrepant results

\begin{tabular}{ccccc}
\hline Sample & Phenotype & Sequencing assay & \% HEXA activity & DNA mutation assay* \\
\hline TS50 & Carrier & No mutation & $48(\mathrm{~A} / \mathrm{C})$ & No mutation \\
TS12 & Carrier & $775 \mathrm{~A} \rightarrow \mathrm{G} \dagger$ & $51(\mathrm{~A} / \mathrm{C})$ & No mutation \\
TS53 & Carrier & No mutation & $51(\mathrm{~A} / \mathrm{C})$ & No mutation \\
\hline
\end{tabular}

$*$ DNA mutation assay includes detection of 7.6-KB DEL, EX1; 739C $\rightarrow$ T; 745C $\rightarrow$ T; 805G $\rightarrow$ A; $1073+1 \mathrm{G} \rightarrow \mathrm{A} ; 1278$ insTATC; and $1421+1 \mathrm{G} \rightarrow \mathrm{C}$.

$\dagger$ Novel mutation; no change in phenotype expected.

$\mathrm{A} / \mathrm{C}$, in affected/carrier range; $\mathrm{N}$, in normal range. 
Table 2. Sensitivity of the enzyme, DNA mutation, and gene sequencing assays

\begin{tabular}{|c|c|c|c|c|c|c|}
\hline Phenotype & Patients no. & $\begin{array}{c}\text { Enzyme } \\
\text { assay (\%) }\end{array}$ & $\begin{array}{l}\text { DNA mutation } \\
\text { assay* }(\%)\end{array}$ & $\begin{array}{c}\text { Enzyme }+ \\
\text { DNA mutation }(\%)\end{array}$ & $\begin{array}{l}\text { Sequencing } \\
\text { assay }(\%)\end{array}$ & $\begin{array}{c}\text { Enzyme }+ \\
\text { sequencing }(\%)\end{array}$ \\
\hline TS disease & 6 & 6/6 (100) & 6/6 (100) & 6/6 (100) & 6/6 (100) & 6/6 (100) \\
\hline TS carrier & 33 & $30 / 33(91)$ & $17 / 33(52)$ & $32 / 33(97)$ & $30 / 33(91)$ & 33/33 (100) \\
\hline TS disease/carrier & 39 & $36 / 39(92)$ & 23/39 (59) & 38/39 (97) & $36 / 39(92)$ & $39 / 39(100)$ \\
\hline Pseudo & 1 & Indeterminate & 1/1 (100) & 1/1 (100) & 1/1 (100) & 1/1 (100) \\
\hline
\end{tabular}

* DNA mutation assay includes detection of 7.6-KB DEL, EX1; 739C $\rightarrow \mathrm{T} ; 745 \mathrm{C} \rightarrow \mathrm{T} ; 805 \mathrm{G} \rightarrow \mathrm{A} ; 1073+1 \mathrm{G} \rightarrow \mathrm{A} ; 1278 \mathrm{insTATC}$; and $1421+1 \mathrm{G} \rightarrow \mathrm{C}$.

Table 3. Enzyme assay discrepant results

\begin{tabular}{clccc}
\hline Sample & Phenotype & \% HEXA activity & DNA mutation assay* & Sequencing assay \\
\hline TS36 & Pseudodeficient & $53(\mathrm{I})$ & $739 \mathrm{C} \rightarrow \mathrm{T}$ & $739 \mathrm{C} \rightarrow \mathrm{T}$ \\
TS38 & Carrier & $55(\mathrm{I})$ & $805 \mathrm{G} \rightarrow \mathrm{A}$ & $805 \mathrm{G} \rightarrow \mathrm{A}$ \\
TS16 & Carrier & $82(\mathrm{~A})$ & No mutation & $533 \mathrm{G} \rightarrow \mathrm{A}$ \\
\hline
\end{tabular}

* DNA mutation assay includes detection of 7.6-KB DEL, EX1; 739C $\rightarrow \mathrm{T} ; 745 \mathrm{C} \rightarrow \mathrm{T} ; 805 \mathrm{G} \rightarrow \mathrm{A} ; 1073+1 \mathrm{G} \rightarrow \mathrm{A} ; 1278$ insTATC; and 1421 + 1G $\rightarrow \mathrm{C}$.

$\mathrm{I}$, in indeterminate range; $\mathrm{N}$, in normal range; $\mathrm{A}$, above normal range.

Table 4. Novel mutations detected by HEXA gene sequencing

\begin{tabular}{cccccc}
\hline Sample & Phenotype & Novel mutation & Predicted protein damage* & Known mutation & $\%$ HEXA activity \\
\hline TS21 & Carrier & $759 \mathrm{G} \rightarrow \mathrm{A}$ V253V & No & TATCins 1278 \\
TS33 & Carrier $\dagger$ & $965 \mathrm{~A} \rightarrow \mathrm{T} \mathrm{D322V}$ & Yes & None & No (A/C) \\
TS32 & Carrier $\dagger$ & $965 \mathrm{~A} \rightarrow \mathrm{T} \mathrm{D322V}$ & Yes & None \\
TS23 & Carrier & $118 \mathrm{Tdel}$ & Yes & None & $49(\mathrm{~A} / \mathrm{C})$ \\
TS12 & Carrier & $775 \mathrm{~A} \rightarrow \mathrm{G} \mathrm{T259A}$ & No & None & $51(\mathrm{~A} / \mathrm{C})$ \\
\hline
\end{tabular}

* PolyPen and SHIFT web-tools were used for protein folding prediction.

$\dagger$ Blood relatives (mother/daughter).

$\mathrm{A} / \mathrm{C}$, in affected/carrier range.

Sensitivity of the gene sequencing assay was substantially higher than that of the DNA common mutation test: $91 \%$ of TSD carriers and affected were correctly classified by gene sequencing versus only $52 \%$ correctly classified by the DNA mutation test (Table 2). The enzyme assay sensitivity and gene sequencing had similar sensitivities (91\%). Of the two cases with indeterminate enzyme results, gene sequencing correctly classified one as a pseudodeficiency carrier and the other as a TSD carrier. In addition, gene sequencing detected a TSD-associated mutation $(533 \mathrm{G} \rightarrow \mathrm{A})$ in a TSD carrier who had an enzyme assay result above the normal range. The $533 \mathrm{G} \rightarrow \mathrm{A}$ mutation is associated with the rare B1 variant phenotype known to overestimate HEXA enzyme activity when a neutral substrate such as MUG is used $(15,16)$. Thus, gene sequencing helped clarify enzyme test results that did not correlate with the phenotype (Table 3). We also tested six Sandhoff carriers for HEXA DNA common mutation and HEXA gene sequencing assays and found no mutation in the HEXA gene. Combined use of the enzyme assay and gene sequencing had the greatest sensitivity (100\%).

Gene sequencing detected four mutations (Table 4) we believe to be novel, because we found no report of them either in the Tay-Sachs mutation database at McGill University (http://www.hexdb.mcgill.ca/?Topic=HEXAdb) or in the PubMed database. A single nucleotide deletion, 118.delT, in exon 1 was found in one carrier. This leads to one nucleotide frame shift resulting in a truncated protein that ends at exon 2 . Therefore, this is most likely a disease-causing mutation. A missense mutation, 965A $\rightarrow \mathrm{T}(\mathrm{D} 322 \mathrm{~V})$, causes an amino acid change from a positively charged aspartic acid to a hydrophobic valine. This is likely to be disease-causing because one of the two individuals with this mutation is an obligate carrier and has a HEXA activity result in the carrier range and no other detected mutation. In addition, PolyPen and SHIFT web-based tools suggest damage at the ligand-binding site that interferes with protein folding $(17,18)$. Another missense mutation, $775 \mathrm{~A} \rightarrow \mathrm{G}$ changes a hydrophilic threonine to a hydrophobic alanine at amino acid position 259 (T259A). It may be a disease-causing mutation because the enzyme activity was in the carrier level (51\% HEXA) and no other TSD-associated mutation was detected. However, the predicted protein folding seems to be minimal according to PolyPen and SHIFT webbased tools. A silent mutation, $759 \mathrm{G} \rightarrow \mathrm{A}(\mathrm{V} 253 \mathrm{~V})$, in exon 7 was detected in a carrier who also has a 1278insTATC allele. Thus, $759 \mathrm{G} \rightarrow \mathrm{A}$ is most likely a benign polymorphism.

\section{DISCUSSION}

The data demonstrate that no single test is capable of identifying all carriers of TSD. The presence of a B1 variant in 33 obligate carriers in this study demonstrates that enzyme testing alone cannot correctly identify all TSD carriers. DNA sequencing alone similarly did not detect three obligate carriers. The combination of Hex A enzyme assay and HEXA gene sequencing detected all obligate carriers, whereas the combination of Hex enzyme assay and HEXA DNA common mutation test detected all but the one carrier with the $\mathrm{B} 1$ allele.

The most cost effective and accurate TSD carrier detection method would be a combination of Hex A enzyme assay with a DNA common mutation test that was expanded to include the B1 allele, because the allele leads to false-negative Hex A 
enzyme analysis. The particular B1 variant $(533 \mathrm{G} \rightarrow \mathrm{A})$ we detected is present in wide geographic and ethnic distribution (19), and its frequency is especially high in Portuguese population, where its prevalence is estimated to be 0.84 per 100,000 live births (20).

DNA sequencing did not detect a Hex A mutation in three TSD carriers. The sequencing method, however, is limited in that it does not identify large deletions or insertions or mutations well into an intronic sequence. Such mutations seem to be relatively rare, though, and our sequencing assay covers all of the known intronic mutations. DNA sequencing is also limited in that the promoter region is not sequenced, but no mutations have been reported in this region thus far. Mutations in genes other than the HEXA gene are not identified, though to date none are known to be associated with the TSD phenotype. Nevertheless, sequencing is not $100 \%$ sensitive, and the cost is prohibitively high for use in population-based carrier screening.

When compared with the HEXA enzyme assay and the DNA common mutation assay, the primary benefit of gene sequencing in this study was to increase overall sensitivity, clarify discrepant results obtained by the other two assays, and identify novel mutations. In our study environment, we compared test results to a "known" phenotype/carrier status; however, this is not the environment in which these assays are used. Is there an appropriate role for HEXA gene sequencing in the clinical environment that seeks to identify carriers and affected individuals?

Multiple authors have recommended common DNA mutation testing for high-risk populations such as the Ashkenazi Jewish population (4). The ACOG Committee on Genetics does not specifically recommend a screening method for high-risk populations, but does recommend use of the enzyme test for individuals in low-risk populations (2). For the nonAshkenazi Jewish population, our data show the HEXA enzyme assay will detect a greater percentage of TSD carriers than a DNA common mutation test. Because the enzyme assay indirectly quantitates HEXA activity, however, ambiguous results are often obtained. HEXA gene sequencing analysis is appropriate for obligate carriers or affected patients whose mutation has not been identified, for carrier detection in spouses of known carriers who have indeterminate enzyme analysis and negative for the common mutation and to resolve diagnostic dilemmas from ambiguous enzyme testing.

In conclusion, we have successfully detected various small mutations in the HEXA gene through gene sequencing and, by combining the HEXA enzyme assay and the HEXA gene sequencing assay, we were able to clarify TSD carrier status in $100 \% 34$ obligate TSD carriers. No single testing modality was able to identify all obligate TSD carriers. This has important implications if TSD population screening is expanded to non-AJ populations.

Acknowledgments. We gratefully acknowledge Patricia M. Vendely for assistance in writing and editing this manuscript.

\section{REFERENCES}

1. Kaback M, Lim-Steele J, Dabholkar D, Brown D, Levy N, Zeiger K 1993 Tay-Sachs disease-carrier screening, prenatal diagnosis, and the molecular era. An international perspective 1970 to 1993 . The International TSD Data Collection Network. JAMA 270:2307-2315

2. ACOG Committee on Genetics 2005 ACOG committee opinion. Number 318 , October 2005. Screening for Tay-Sachs disease. Obstet Gynecol 106:893-894

3. O'Brien JS, Okada S, Chen A, Fillerup DL 1970 Tay-sachs disease. Detection of heterozygotes and homozygotes by serum hexosaminidase assay. N Engl J Med 283:15-20

4. Bach G, Tomczak J, Risch N, Ekstein J 2001 Tay-Sachs screening in the Jewish Ashkenazi population: DNA testing is the preferred procedure. Am J Med Genet 99:70-75

5. Navon R, Mark Z, Mashiah S, Padeh B 1973 Determination of Tay-Sachs genotypes in pregnant women. Clin Genet 4:286-287

6. Nitowsky HM, Davis J, Nakagawa S, Fox D 1979 Human hexosaminidase isozymes. IV. Effects of oral contraceptive steroids on serum hexosaminidase activity. Am J Obstet Gynecol 134:642-647

7. Cao Z, Natowicz MR, Kaback MM, Lim-Steele JS, Prence EM, Brown D, Chabot T, Triggs-Raine BL 1993 A second mutation associated with apparent betahexosaminidase A pseudodeficiency: identification and frequency estimation. Am J Hum Genet 53:1198-1205

8. Triggs-Raine BL, Mules EH, Kaback MM, Lim-Steele JS, Dowling CE, Akerman BR, Natowicz MR, Grebner EE, Navon R, Welch JP, Greenberg CR, Thomas GH, Gravel RA 1992 A pseudodeficiency allele common in non-Jewish Tay-Sachs carriers: implications for carrier screening. Am J Hum Genet 51:793-801

9. Kytzia HJ, Sandhoff K 1985 Evidence for two different active sites on human beta-hexosaminidase A. Interaction of GM2 activator protein with betahexosaminidase A. J Biol Chem 260:7568-7572

10. Rozenberg R, Kok F, Burin MG, Sa Miranda MC, Vasques C, Henriques-Souza AM, Giugliani R, Vainzof M, Pereira LV 2006 Diagnosis and molecular characterization of non-classic forms of Tay-Sachs disease in Brazil. J Child Neurol 21:540-544

11. Grebner EE, Tomczak J 1991 Distribution of three alpha-chain beta-hexosaminidase A mutations among Tay-Sachs carriers. Am J Hum Genet 48:604-607

12. Triggs-Raine BL, Feigenbaum AS, Natowicz M, Skomorowski MA, Schuster SM, Clarke JT, Mahuran DJ, Kolodny EH, Gravel RA 1990 Screening for carriers of Tay-Sachs disease among Ashkenazi Jews. A comparison of DNA-based and enzyme-based tests. N Engl J Med 323:6-12

13. Huang D, Chen C, Sun W, Strom CM, Bender RA 2004 High-throughput gene sequencing assay development for hereditary nonpolyposis colon cancer. Clin Colorectal Cancer 4:275-279

14. Strom CM, Janeczko RA, Anderson B, Redman J, Quan F, Buller A, McGinniss MJ, Sun WM 2005 Technical validation of a multiplex platform to detect thirty mutations in eight genetic diseases prevalent in individuals of Ashkenazi Jewish descent. Genet Med 7:633-639

15. Tutor JC 2004 Biochemical characterization of the GM2 gangliosidosis B1 variant. Braz J Med Biol Res 37:777-783

16. Whitley CB, Anderson RA, McIvor RS 1992 Heterozygosity for the "DN allele" (G533-greater than A) of the beta-hexosaminidase alpha subunit gene identified by direct DNA sequencing in a family with the B1 variant of GM2-gangliosidosis. Neuropediatrics 23:96-101

17. Ng PC, Henikoff S 2006 Predicting the effects of amino acid substitutions on protein function. Annu Rev Genomics Hum Genet 7:61-80

18. Ramensky V, Bork P, Sunyaev S 2002 Human non-synonymous SNPs: server and survey. Nucleic Acids Res 30:3894-3900

19. Tanaka A, Ohno K, Suzuki K 1988 GM2-gangliosidosis B1 variant: a wide geographic and ethnic distribution of the specific beta-hexosaminidase alpha chain mutation originally identified in a Puerto Rican patient. Biochem Biophys Res Commun 156:1015-1019

20. Teixeira CA, Sena-Esteves M, Lopes L, Sa Miranda MC, Ribeiro MG 2001 Retrovirus-mediated transfer and expression of beta-hexosaminidase alpha-chain cDNA in human fibroblasts from G(M2)-gangliosidosis B1 variant. Hum Gene Ther 12:1771-1783 